\title{
Moment evolution across the ferromagnetic phase transition of giant magnetocaloric $(\mathrm{Mn}, \mathrm{Fe})_{2}(\mathrm{P}, \mathrm{Si}, \mathrm{B})$ compounds
}

\author{
H. Yibole,,${ }^{1, *}$ F. Guillou, ${ }^{1}$ L. Caron, ${ }^{1}$ E. Jiménez, ${ }^{2}$ F. M. F. de Groot,${ }^{3}$ P. Roy, ${ }^{4}$ R. de Groot,${ }^{4}$ N. H. van Dijk,,${ }^{1}$ and E. Brück ${ }^{1}$ \\ ${ }^{1}$ FAME, TU Delft, Mekelweg 15, 2629JB Delft, The Netherlands \\ ${ }^{2}$ European Synchrotron Radiation Facility, 71 Avenue des Martyrs CS40220, F-38043 Grenoble Cedex 09, France \\ ${ }^{3}$ Inorganic Chemistry and Catalysis, Utrecht University, 3584 CG Utrecht, The Netherlands \\ ${ }^{4}$ Electronic Structure of Materials, Faculty of Sciences, Radboud University, 6525 AJ Nijmegen, The Netherlands
}

(Received 10 October 2014; revised manuscript received 19 December 2014; published 23 January 2015)

\begin{abstract}
A strong electronic reconstruction resulting in a quenching of the Fe magnetic moments has recently been predicted to be at the origin of the giant magnetocaloric effect displayed by $\mathrm{Fe}_{2} \mathrm{P}$-based materials. To verify this scenario, $\mathrm{X}$-ray magnetic circular dichroism experiments have been carried out at the $L$ edges of $\mathrm{Mn}$ and Fe for two typical compositions of the $(\mathrm{Mn}, \mathrm{Fe})_{2}(\mathrm{P}, \mathrm{Si}, \mathrm{B})$ system. The dichroic absorption spectra of $\mathrm{Mn}$ and $\mathrm{Fe}$ have been measured in the vicinity of the first-order ferromagnetic transition. The experimental spectra are compared with first-principles calculations and charge-transfer multiplet simulations in order to derive the magnetic moments. Even though signatures of a metamagnetic behavior are observed either as a function of the temperature or the magnetic field, the similarity of the Mn and Fe moment evolution suggests that the quenching of the Fe moment is weaker than previously predicted.
\end{abstract}

DOI: 10.1103/PhysRevB.91.014429

PACS number(s): 75.30.Sg, 75.40.-s, 75.50.Bb

\section{INTRODUCTION}

In the field of functional magnetic materials, compounds exhibiting a first-order magnetic phase transition (FOMT) have recently received a large interest due to their potential applications. Systems that undergo a discontinuous phase transformation have especially been studied for their magnetocaloric effect (MCE). One of the most promising MCE applications is magnetic cooling, which overturns the use of greenhouse or ozone-depleting refrigerant gases, while it potentially has a better energy efficiency than usual cooling methods [1-3]. To observe a particularly large magnetocaloric effect, advantage should be taken from the latent heat displayed by the first-order magnetic transition leading to a giant magnetocaloric effect (GMCE). In the last decade several families of MCE materials have been discovered: FeRh [4], $\mathrm{Gd}_{5} \mathrm{Si}_{2} \mathrm{Ge}_{2}$ [5], $\mathrm{Mn}(\mathrm{As}, \mathrm{Sb})$ [6], $\mathrm{La}(\mathrm{Fe}, \mathrm{Si})_{13}$ and its hydrides [7,8], and $\operatorname{MnFe}(\mathrm{P}, X)(X=\mathrm{As}, \mathrm{Ge}, \mathrm{Si}, \mathrm{B})$ [9-13]. The understanding of the origin of the FOMT in these various materials is still limited. Especially the mechanism at the basis of the interplay between the magnetic/electronic/structural degrees of freedom that leads to a simultaneous change of all these parameters at the FOMT requires more insight and is crucial for further development of advanced magnetocaloric materials. As pointed out by several studies, the latent heat is the key parameter driving the GMCE properties [13-16]. As an example, it has recently been reported that the substitution of a small amount of $\mathrm{P}$ by $\mathrm{B}$ in $\mathrm{MnFe}(\mathrm{P}, \mathrm{Si})$ materials results in a decrease of the latent heat, resulting in better magnetocaloric properties at intermediate magnetic fields [13].

Many efforts have recently been made to understand the FOMT in $\mathrm{Fe}_{2} \mathrm{P}$-based $(\mathrm{Mn}, \mathrm{Fe})_{2}(\mathrm{P}, \mathrm{Si}, \mathrm{B})$ alloys. In this hexagonal system (space group $P \overline{6} 2 m$ ), the Fe and $\mathrm{Mn}$ atoms preferentially occupy the tetragonal $3 f$ site and the pyramidal $3 g$ site, respectively $[17,18]$. When both sites are

\footnotetext{
*Yibole@tudelft.nl
}

occupied by iron, a clear distinction is found between the low-moment $3 f$ site and the high-moment $3 g$ site [17]. The FOMT of these materials has been extensively characterized; in particular, the structural evolution across the FOMT is now well documented [18-23]. All these studies support the magnetoelastic nature of the first-order ferro-to-paramagnetic transition, which leads to a discontinuity in the ratio of the cell parameters $(c / a)$, but keeps the hexagonal structure unmodified with a negligible volume change. Recently, it has been proposed that the key parameter at the origin of GMCE is a strong electronic reconstruction at the FOMT. Electronic structure calculation on $\mathrm{MnFeP}_{0.5} \mathrm{Si}_{0.5}$ [11] predicts a change in hybridization between the $3 f$ site, occupied by iron, and the surrounding nonmetallic elements, which is expected to result in a reduction of the $\mathrm{Fe}(3 f)$ local moment from $1.54 \mu_{\mathrm{B}}$ in the ferromagnetic phase to a value of only $0.003 \mu_{\mathrm{B}}$ in the paramagnetic phase, while the moments on the $3 g$ site $(\mathrm{Mn})$ are almost unaffected [11]. Even though $\mathrm{Fe}_{2} \mathrm{P}$ presents a FOMT one order of magnitude weaker than $\mathrm{MnFe}(\mathrm{P}, \mathrm{Si})$ alloys, a relatively similar mechanism was proposed by Yamada and Terao in the parent material [24]. Within a Ginzburg-Landau model, the loss of long-range magnetic order at the FOMT was ascribed to a cooperative effect between the $3 f$ and $3 g$ sites, resulting in a significant reduction of the local $3 f$ moments at $T_{\mathrm{C}}$. These predictions have recently been revisited to take into account the influence of substitutional elements on the nonmetallic site of $\mathrm{Fe}_{2} \mathrm{P}$ [25].

The evolution of the magnetic moments in $\mathrm{Mn}_{1.25} \mathrm{Fe}_{0.7} \mathrm{P}_{0.5} \mathrm{Si}_{0.5}$, observed by neutron diffraction, seem to support this Fe-quenching scenario [18]. In particular, a reduction of the local $3 f$ magnetic moment in the ferromagnetic phase is observed when $T_{\mathrm{C}}$ is approached, which is compatible with a loss of the local magnetic moment at the FOMT. One of the aims of the present study is to pursue this analysis towards the paramagnetic regime. To test the predicted disappearance of the Fe moment in the paramagnetic phase, X-ray magnetic circular dichroism (XMCD) as a function of temperature and magnetic field 
has been measured. This method allows one to probe the evolution of the local moment both in the ferromagnetic and paramagnetic phases. This is in contrast to neutron diffraction, where site-specific moments are probed. This study has been carried out on two prototypical $\mathrm{MnFe}(\mathrm{P}, \mathrm{Si}, \mathrm{B})$ materials: (i) $\mathrm{MnFe}_{0.95} \mathrm{P}_{0.582} \mathrm{~B}_{0.078} \mathrm{Si}_{0.34}$ (composition $A$ ), which exhibits a good MCE performance, with a $\mathrm{Mn}: \mathrm{Fe}$ ratio close to 1 , where Fe fully occupies the $3 f$ site and $\mathrm{Mn}$ the $3 g$ site; (ii) a Mn-rich material $\mathrm{Mn}_{1.25} \mathrm{Fe}_{0.70} \mathrm{P}_{0.50} \mathrm{~B}_{0.01} \mathrm{Si}_{0.49}$ (composition $B$ ), comparable with the composition used for the previous studies of the $\mathrm{MnFe}(\mathrm{P}, \mathrm{Si})$ system $[12,18,26]$.

\section{EXPERIMENTAL METHODS}

Polycrystalline samples of $\mathrm{MnFe}_{0.95} \mathrm{P}_{0.582} \mathrm{~B}_{0.078} \mathrm{Si}_{0.34}$ and $\mathrm{Mn}_{1.25} \mathrm{Fe}_{0.70} \mathrm{P}_{0.50} \mathrm{~B}_{0.01} \mathrm{Si}_{0.49}$ were prepared according to the same method used in previous studies [13]. Note that the ball-milling and sintering are both done in argon atmosphere. The as-synthesized samples were cycled five times across the FOMT prior to the measurements. Magnetization measurements were carried out in a magnetometer (Quantum Design MPMS 5XL) equipped with a superconducting quantum interference device (SQUID) and the reciprocating sample option.

The XMCD measurements were collected at the ID08 beamline of the European Synchrotron Radiation Facility in Grenoble, France. The measurements were taken by tuning the energy at both the $\mathrm{Mn}$ and $\mathrm{Fe} L_{2,3}$ edges ( $2 p \rightarrow 3 d$ transition). The $\mathrm{x}$-ray absorption spectra were recorded using the totalelectron yield (TEY) mode, and normalized to the intensity of the incident beam. The sample temperature was regulated in the temperature range from 230 to $330 \mathrm{~K}$. The $\mathrm{x}$-ray absorption (XAS) spectra correspond to the sum of positive $\left(\mu^{+}\right)$and negative $\left(\mu^{-}\right)$absorption signals, while the XMCD spectrum is calculated from the difference between $\mu^{+}$and $\mu^{-}$. The bulk samples (circular disks with a diameter of $10 \mathrm{~mm}$ and a thickness of $2 \mathrm{~mm}$ ) were placed in an ultrahigh vacuum (UHV) system equipped with a $5 \mathrm{~T}$ split coil superconducting magnet. The incident $\mathrm{x}$-ray beam and magnetic field are parallel to each other and oriented perpendicular to the sample surface. The pellets were scrapped in situ with a diamond file in the preparation chamber before the measurements. In order to reduce the occurrence of systematic errors, all measurements were performed for two directions of the applied magnetic field, along and opposite to the incident X-ray beam.

In order to simulate the $2 p \mathrm{XAS}$ and XMCD, the spectra were modeled using the ligand field multiplet theory, where we used the CTM4XAS interface version of the programs [27-29]. This approach takes into account all the electronic Coulomb interactions as well as the spin-orbit coupling on any electronic open shell and treats the geometrical environment of the absorbing atom through the crystal-field potential. The spectrum is calculated as the sum of all possible transitions for an electron excited from the $2 p$ level to a $3 d$ level. The $2 p 4 s$ transitions are omitted as they have only small intensity. In the simplest formulation, a pure $3 d^{n}$ configuration is attributed to the $3 d$ transition ions in the ground state and transitions between the $2 p^{6} 3 d^{n}$ ground state and the $2 p^{5} 3 d^{n+1}$ final excited state are calculated. The interelectronic repulsions are introduced through Slater-Condon integrals, $F_{d d}^{2}, F_{d d}^{4}$ and the $3 d$ spin-orbit coupling $\left(\xi_{3 d}\right)$ for the initial state and $F_{d d}^{2}$, $F_{d d}^{4}, F_{p d}^{2}, G_{p d}^{1}, G_{p d}^{3}, \xi_{3 d}$, and $\xi_{2 p}$ for the final state. Atomic spin-orbit values and Slater-Condon integrals have been used, where the Slater-Condon integrals are calculated via a $80 \%$ reduction of the Hartree-Fock values. The surrounding of the metal ion is represented by an octahedral crystal-field potential, parametrized by the parameter $10 D q$ that defines the energy difference between the $t_{2 g}$ and $e_{g}$ orbitals. A molecular field $\left(\mu_{\mathrm{B}} H\right)$ of $0.02 \mathrm{eV}$ is added along the $z$ direction to describe the magnetic order.

The DFT calculations were done by using WIEN2K [30], which employs the full potential linearized augmented plane wave method [31]. The calculations were performed within a scalar relativistic mode. The nonoverlapping atomic sphere radii were taken as 2.23, 2.45, 2.08, and 1.98 a.u. for $\mathrm{Fe}$, $\mathrm{Mn}, \mathrm{Si}$, and $\mathrm{P}$, respectively. The Brillouin zone integration was performed with the tetrahedron method with $72 k$ points within the irreducible Brillouin zone (IBZ). Exchange interactions were taken into account using generalized gradient approximation by Perdew, Burke, and Ernzerhof [32]. To model the XMCD spectra, we first converged the spin-polarized calculation. Since the calculation of optical properties requires a dense mesh of eigenvalues and eigenvectors [33], we chose a $112 k$-point mesh in the IBZ. Then the spin-orbit calculation is performed via a second variational scheme with the direction of magnetization specified along the crystallographic $z$ axis. Core states were treated fully relativistically. So states with orbital angular momentum $l \neq 0$, show splitting due to spin-orbit interaction. To obtain the XMCD peaks at the correct energies, we considered the Slater transition state. So, $1 / 2$ electron was removed either from the $2 P_{3 / 2}$ or $2 P_{1 / 2}$ state and added to the valence states. The energy and the charge were converged to $0.0005 \mathrm{Ry}$ and 0.005 electrons, respectively. Then the momentum matrix elements between the specific core states and the conduction states were calculated and finally the integration over the Brillouin zone was done.

\section{RESULTS AND DISCUSSION}

The temperature dependence of the magnetization for $\mathrm{MnFe}_{0.95} \mathrm{P}_{0.582} \mathrm{~B}_{0.078} \mathrm{Si}_{0.34}$ (composition $A$ ) and $\mathrm{Mn}_{1.25} \mathrm{Fe}_{0.70} \mathrm{P}_{0.50} \mathrm{~B}_{0.01} \mathrm{Si}_{0.49}$ (composition $B$ ) are plotted in Figs. 1(a) and 1(b). In agreement with previous reports $[11,13]$, the Curie temperatures derived from $M_{\mathrm{B}}(T)$ magnetization measurements in $2 \mathrm{~T}$, are $T_{\mathrm{C}}=295$ and $298 \mathrm{~K}$ for compositions $A$ and $B$, respectively. A limited thermal hysteresis $(\sim 2 \mathrm{~K})$ is noticeable between the $M_{\mathrm{B}}(T)$ upon warming and upon cooling for composition $A$. For composition $B$ the hysteresis is negligible. In addition, the magnetization jump appears to be broader in $B$ than in $A$. Both features point towards a weaker first-order character in material $B$ than in $A$. This tendency is supported by the isothermal magnetization curves presented in Figs. 1(c) and 1(d) for materials $A$ and $B$, respectively. The $M_{\mathrm{T}}(B)$ curves for sample $A$ with $\mathrm{Mn}: \mathrm{Fe}$ ratio close to 1 show a clear $\mathrm{S}$ shape in the vicinity of $T_{\mathrm{C}}$, which confirms the occurrence of a FOMT. This metamagnetic behavior is far less pronounced for material $B$. The Arrot plots in Figs. 1(e) and 1(f) indicate that sample $B$ lays at the boundary where the FOMT turns into a 

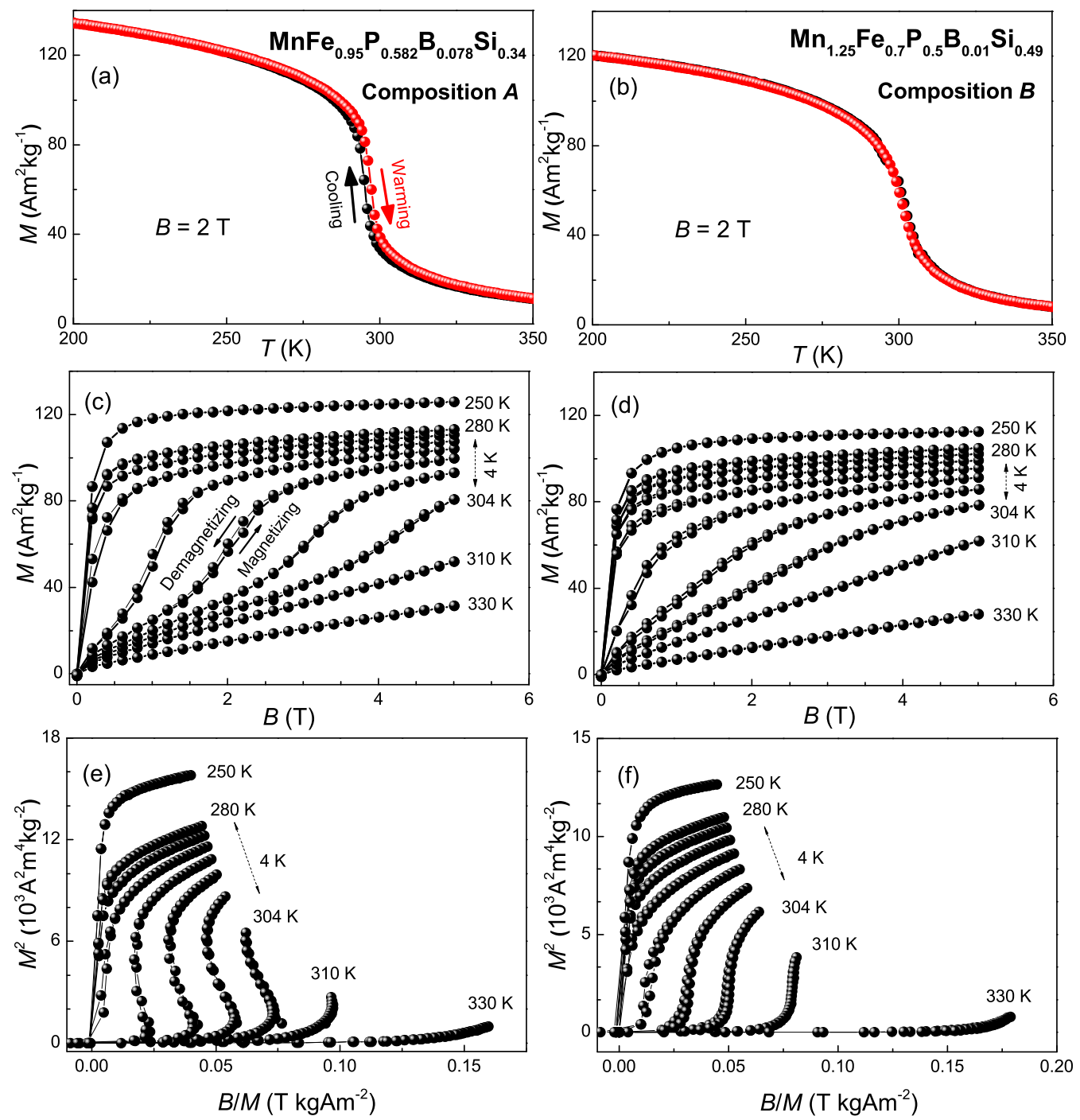

FIG. 1. (Color online) Temperature dependence of the magnetization in a field of $2 \mathrm{~T}$ for $\mathrm{MnFe}_{0.95} \mathrm{P}_{0.582} \mathrm{~B}_{0.078} \mathrm{Si}_{0.34}$ (composition $A$ ) (a) and $\mathrm{Mn}_{1.25} \mathrm{Fe}_{0.70} \mathrm{P}_{0.50} \mathrm{~B}_{0.01} \mathrm{Si}_{0.49}$ (composition $B$ ) (b), the magnetic isotherms in the vicinity of the transition temperature for composition $A$ (c), and composition $B(\mathrm{~d})$, and Arrot plots obtained from increasing field magnetization isotherms in the vicinity of the transition temperature for composition $A$ (e) and composition $B$ (f).

continuous transition. The two examples have similar Curie temperatures, but exhibit different transitional behaviors.

Figure 2 shows the experimental XAS and XMCD spectra of composition $A$ at $L_{2,3}$ edges of $\mathrm{Mn}$ and $\mathrm{Fe}$. The spectra were obtained in the ferromagnetic state $(T=250 \mathrm{~K})$ and in the paramagnetic state $(T=330 \mathrm{~K})$ at an applied magnetic field of $4 \mathrm{~T}$. There is almost no difference between the XAS spectra of the ferromagnetic and paramagnetic states [see Fig. 2(a)]. For Mn, the absorption maxima are located at about 639.6 and $650.8 \mathrm{eV}$ for the $L_{3}$ and $L_{2}$ edges, respectively. On the high-energy wing of $L_{3}$, one can observe two satellite peaks: one at about $1 \mathrm{eV}$ and another (hardly visible) at about $3.5 \mathrm{eV}$ above the maximum of $L_{3}$. At the $L_{2}$ edges, there is a tendency towards a peak splitting into two separate peaks. The two peaks are also present in the multiplet calculation of $\mathrm{Mn}$, regarded as a $4 s^{2} 3 d^{5}$ system. Due to band dispersion effects these atomic transitions are broadened in intermetallic and covalent systems. Depending on the degree of broadening the peaks can be distinguishable in experimental spectra, where
$\mathrm{Mn}$ is more localized and expected to have more visible features [28,34]. Similar features were also observed for the Mn spectra of some intermetallic alloys and thin films [35-37]. This more localized character of the $3 g$ site in $\mathrm{Fe}_{2} \mathrm{P}$-based materials has already been pointed out by several theoretical and experimental studies [11,25,38]. However, at this stage one cannot rule out a few other possibilities for the origin of the peak splitting. Although the sample surface was prepared in situ in UHV, one has to keep in mind that a partial oxidation of the surface cannot be totally discarded, especially when measuring in TEY mode (the probing depth is only several $\mathrm{nm})$. In addition, there may be an antisite effect, i.e., a limited amount of Mn site on the $3 f$ tetrahedral site instead of the $3 g$ site with a pyramidal environment [18]. The XAS line shapes of Fe, shown in Fig. 2(b), exhibit relatively broad absorption peaks. The only additional feature is a small peak that appears on the high-energy site of the $L_{3}$ edge, positioned at about $+1.1 \mathrm{eV}$. It is worth noting that for both the Fe and Mn XAS spectra, no energy shifts are observed when the temperature 

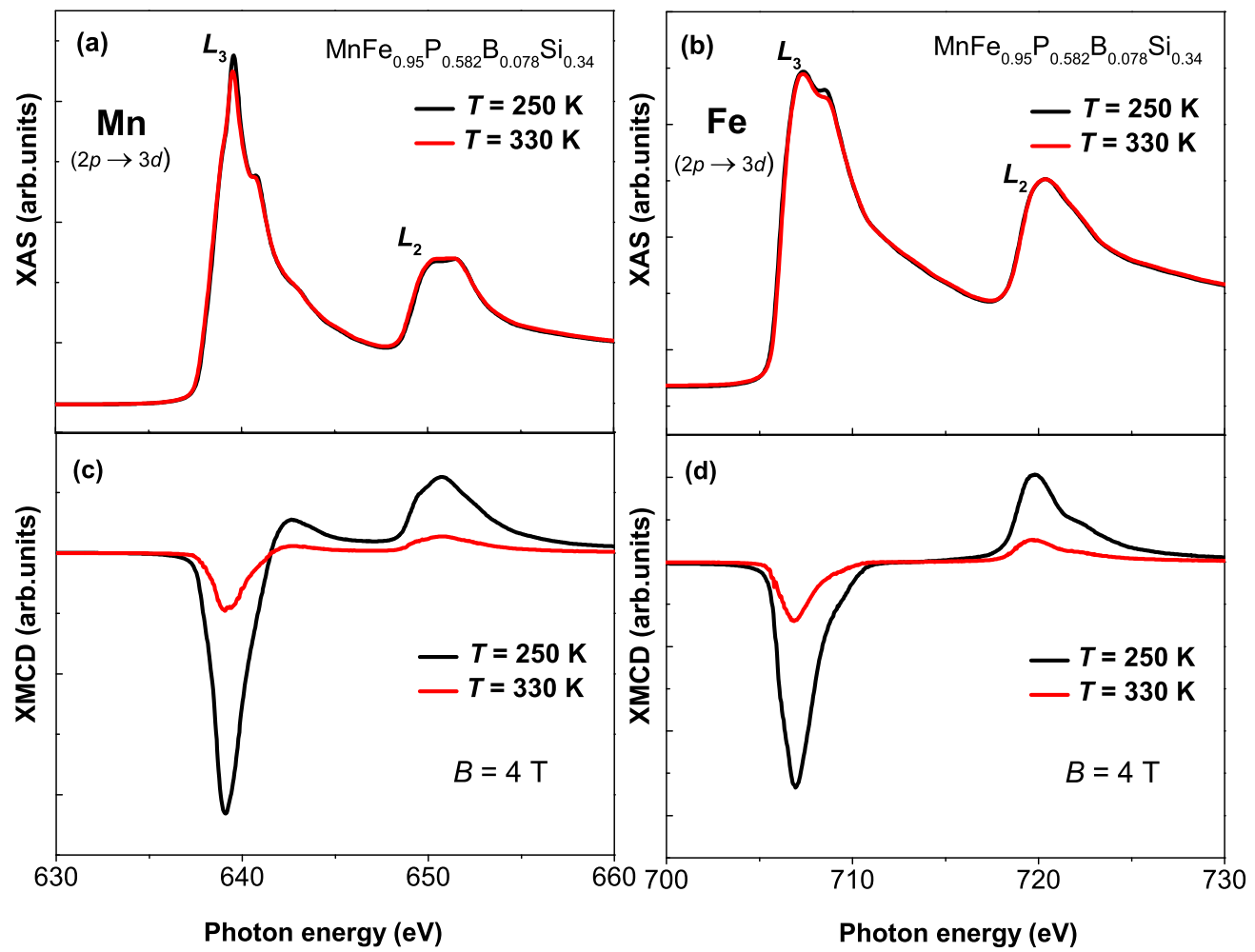

FIG. 2. (Color online) XAS and XMCD spectra for $\mathrm{MnFe}_{0.95} \mathrm{P}_{0.582} \mathrm{~B}_{0.078} \mathrm{Si}_{0.34}$ (composition $A$ ) measured at the Mn- $L_{2,3}$ edge [left panel, (a) and (c)], and $\mathrm{Fe}-L_{2,3}$ edge [right panel, (b) and (d)]. Black and red spectra are measured at $250 \mathrm{~K}$ (ferromagnetic state) and $330 \mathrm{~K}$ (paramagnetic state), under an applied magnetic field of $4 \mathrm{~T}$, respectively.

is changed from the ferromagnetic to the paramagnetic state. This implies that no significant valence change takes place across the phase transition.

Figures 2(c) and 2(d) show the XMCD spectra for sample $A$. The intensity of both the Mn and Fe XMCD are clearly reduced in the paramagnetic state $(330 \mathrm{~K})$, while in both cases the spectral shape remains unmodified. Moreover, the polarity of the XMCD of $\mathrm{Mn}$ and $\mathrm{Fe}$ is the same, which indicates a parallel alignment of the spin moments of $\mathrm{Mn}$ and Fe. This is in good agreement with the neutron diffraction results for $\mathrm{MnFe}(\mathrm{P}, \mathrm{Si})$ compounds in which the spins on the $3 f$ and $3 g$ sites are aligned in parallel in the $a b$ plane [18]. For the Mn spectrum, there is a pronounced positive shoulder on the high-energy side of the $L_{3}$ edge, whose intensity approaches zero before the $L_{3}$ peak. This positive shoulder at the vicinity of $L_{3}$ of the Mn spectrum was also found in atomic calculations for $\mathrm{Mn} 4 s^{2} 3 d^{5}$ states [39]. The contribution at $3.5 \mathrm{eV}$ above $L_{3}$, which is visible in both the XMCD and the XAS signal, is considered to belong to the main phase and to be an intrinsic property of the material. This is a signature of the $j j$ mixing often observed in light $3 d$ elements and will be discussed in detail hereafter. Unlike the anomaly at $L_{3}+3.5 \mathrm{eV}$, the additional peaks at $L_{3}+1 \mathrm{eV}$ in the XAS spectrum of both $\mathrm{Fe}$ and $\mathrm{Mn}$, do not correspond to any feature in the XMCD signal. This suggests that oxidation is the origin of these two satellite peaks at $+1 \mathrm{eV}$ on both the $\mathrm{Mn}$ and $\mathrm{Fe}$ edges. In the case of $\mathrm{Mn}_{1.25} \mathrm{Fe}_{0.70} \mathrm{P}_{0.50} \mathrm{~B}_{0.01} \mathrm{Si}_{0.49}$ (composition $B$ ), the spectral feature of XAS and XMCD spectra of the Mn- and Fe- $L_{2,3}$ edge are shown in Fig. 3. The spectral features for composition $B$ are comparable to that of composition $A$.
Figures 4(a) and 4(b) show the field dependence of the XMCD spectra for $\mathrm{Mn}$ and $\mathrm{Fe}$ at a temperature of $292 \mathrm{~K}$, just above the transition temperature $T_{\mathrm{C}}=288 \mathrm{~K}$ (in zero magnetic field). As anticipated, the XMCD intensity increases with the applied magnetic field, reflecting a gain in the average projection of the magnetic moment with the field. From $B=0$ to $0.75 \mathrm{~T}$, there is a rapid appearance of a sizable XMCD signal, which can be ascribed to the orientation of magnetic domains. Above $0.75 \mathrm{~T}$, one can see a slower increase of the XMCD signal with an approach to a saturation above $3 \mathrm{~T}$. This behavior corresponds to a metamagnetic transition. The critical field $\left(B_{\mathrm{C}}\right)$ is reasonably in line with the $B_{\mathrm{C}} \approx 1.25 \mathrm{~T}$ derived from the $M_{\mathrm{T}}(B)$ curves presented in Fig. 1(c). Comparing Figs. 2 and 4 highlights the similarity between crossing the paramagneticferromagnetic transition by decreasing the temperature or by increasing the field in the present $(T, B)$ range. For both the temperature- and field-induced FOMT, the shape of the XMCD (and XAS) at the Mn and Fe edge is not affected by the change in magnetic field and temperature.

The line shapes of the XAS and XMCD spectra are strongly dependent on the electronic configuration of the probed atoms. In order to analyze the spectral features of the XAS and XMCD spectra in detail, charge transfer (CT) multiplet calculations [27-29] and density functional theory (DFT) calculations were carried out. In Fig. 5, we compare the experimental data at magnetic saturation for composition $A$ with the spectra obtained from the CT and DFT calculations. The DFT calculations can ideally provide a good single-particle (itinerant model) description of the chemical bonds, while the multiplet calculation provides a reliable multiconfigurational 

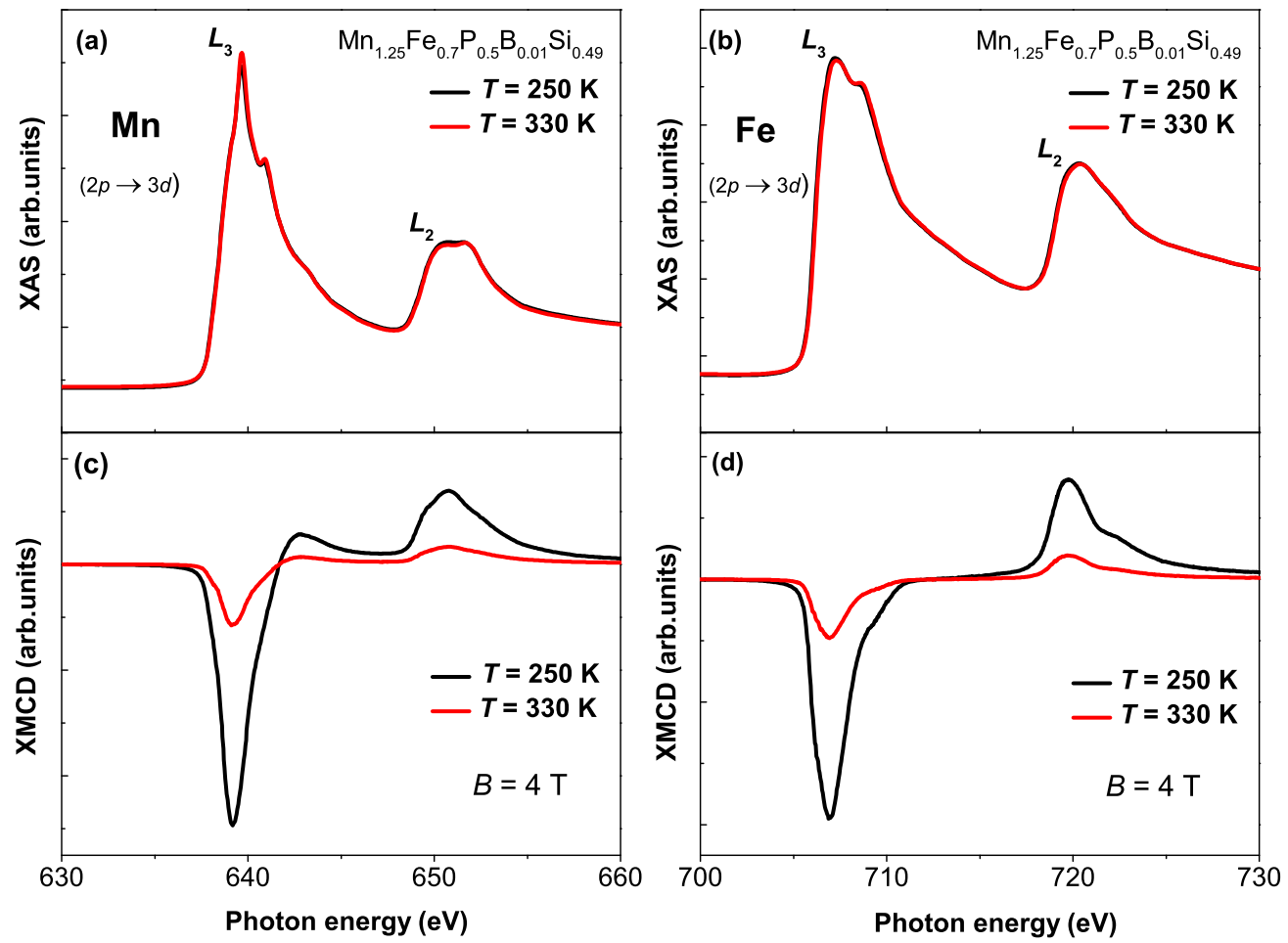

FIG. 3. (Color online) XAS and XMCD spectra for $\mathrm{Mn}_{1.25} \mathrm{Fe}_{0.70} \mathrm{P}_{0.50} \mathrm{~B}_{0.01} \mathrm{Si}_{0.49}$ (composition $B$ ) measured at the Mn- $L_{2,3}$ edge [left panel, (a) and (c)], and $\mathrm{Fe}-L_{2,3}$ edge [right panel, (b) and (d)]. Black and red spectra are measured at $250 \mathrm{~K}$ (ferromagnetic state) and $330 \mathrm{~K}$ (paramagnetic state), under an applied magnetic field of $4 \mathrm{~T}$, respectively.
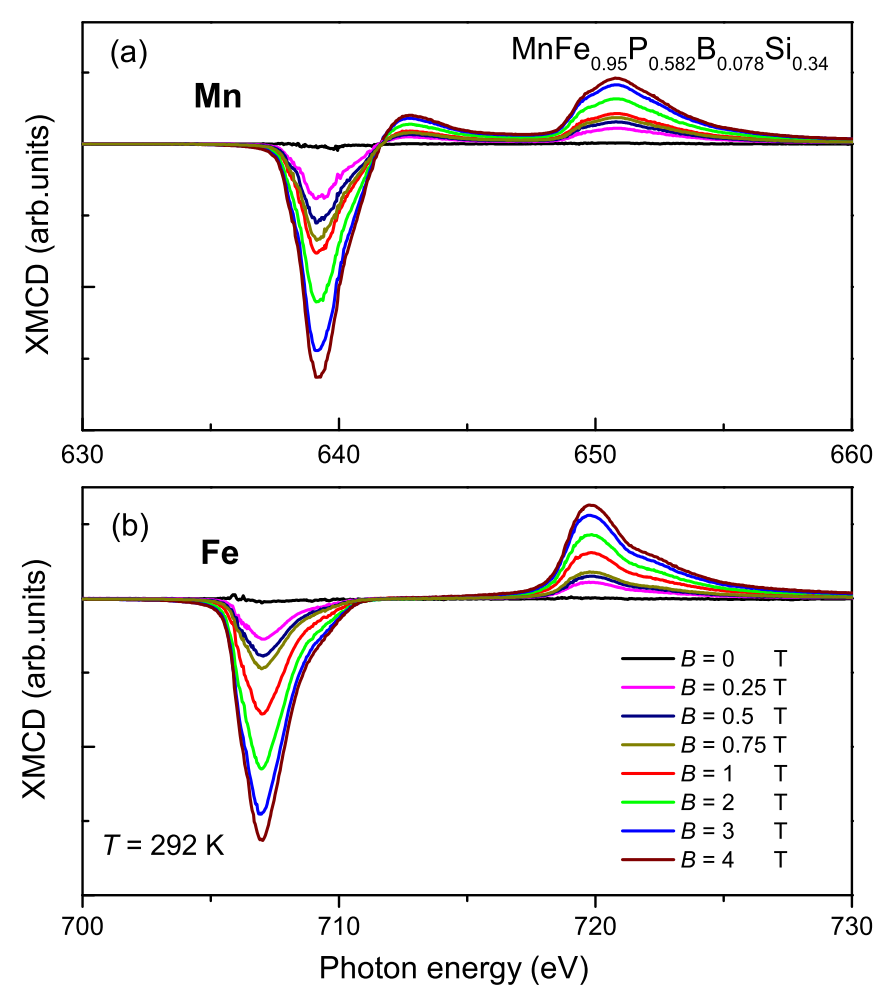

FIG. 4. (Color online) The field dependence of the XMCD spectra for $\mathrm{Mn}-L_{2,3}$ (a) and $\mathrm{Fe}-L_{2,3}$ (b) at a temperature of $292 \mathrm{~K}$ for $\mathrm{MnFe}_{0.95} \mathrm{P}_{0.582} \mathrm{~B}_{0.078} \mathrm{Si}_{0.34}$ (composition $A$ ). The temperature of $292 \mathrm{~K}$ is just above the magnetic transition at zero field of $T_{\mathrm{C}}=288 \mathrm{~K}$ $(B=0 \mathrm{~T})$. description of the final states and their spin-orbit coupling. In the present CT calculations, the spectra were modeled by the $2 p^{6} 3 d^{5} \rightarrow 2 p^{5} 3 d^{6}(\mathrm{Mn})$ and $2 p^{6} 3 d^{6} \rightarrow 2 p^{5} 3 d^{7}(\mathrm{Fe})$ transition in an octahedral $O_{\mathrm{h}}$ symmetry, considering a crystal-field splitting of $10 \mathrm{Dq}=0.2 \mathrm{eV}$ for $\mathrm{Mn}$ and $10 \mathrm{Dq}=1.0 \mathrm{eV}$ for Fe. To account for configuration interaction effects, the Slater integrals were reduced to $80 \%$ of their Hartree-Fock values. The effect of exchange splitting was taken into account by setting the magnetic splitting parameter to $20 \mathrm{meV}$. The final state charge-transfer energy $\Delta+U_{\mathrm{dd}}-U_{\mathrm{pd}}$ has been used with a fixed difference of $U_{\mathrm{pd}}-U_{\mathrm{dd}}=1 \mathrm{eV}$, where $\Delta$ is the charge-transfer energy, $U_{\mathrm{dd}}$ is the Hubbard $U$ correlation energy, and $U_{\mathrm{pd}}$ is the core-hole potential [40]. Each spectrum is broadened with a Lorentzian broadening of $0.2 \mathrm{eV}(0.4 \mathrm{eV})$ for $L_{3}\left(L_{2}\right)$ and a Gaussian broadening of $0.5 \mathrm{eV}$ to approximately account for lifetime and resolution effects. The XMCD was derived from DFT calculations according to the method described in Sec. II.

These two computational methods are compared to the experimental XAS and XMCD data in Figs. 5(a) [5(c)] and 5(b) [5(d)] for the $\mathrm{Mn}$ and $\mathrm{Fe}$ edges, respectively. Generally speaking, there is a decent agreement between the experimental spectra and the calculations. One can see that the overall spectral features are reproduced fairly well by both calculation methods. The energy positions of the main peaks of the two calculated spectra agree with that of experimental spectra. Besides, the main and satellite features are reproduced correctly within $1 \mathrm{eV}$ and the calculated intensity distributions are rather realistic [see Figs. 5(a) and 5(b)]. However, one observes that the relative intensity ratio $L_{2} / L_{3}$, which depends mainly on the projection of the total moment, shows some 

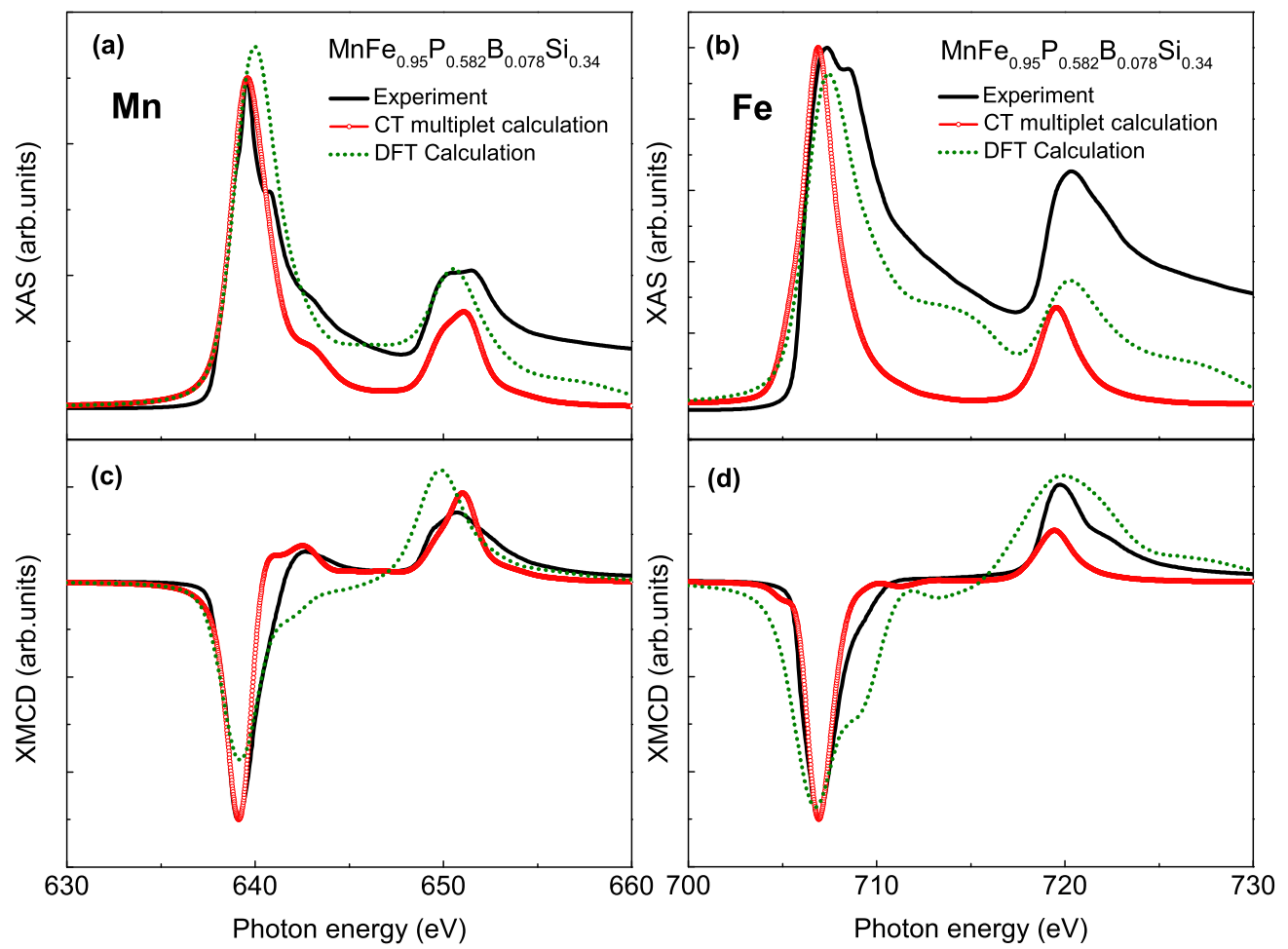

FIG. 5. (Color online) Comparison of the measured, charge-transfer multiplet calculation and DFT calculation for XAS and XMCD spectra at the $\mathrm{Mn}-L_{2,3}\left[(\mathrm{a})\right.$ and (c)] and Fe- $L_{2,3}\left[(\mathrm{~b})\right.$ and (d)] edge, for $\mathrm{MnFe}_{0.95} \mathrm{P}_{0.582} \mathrm{~B}_{0.078} \mathrm{Si}_{0.34}$ (composition $A$ ). Black full line shows the measured spectra at $250 \mathrm{~K}, 4 \mathrm{~T}$. Red symbols and green dots show the spectra from the multiplet and DFT calculations, as described in the text.

deviation for both calculations [see Figs. 5(c) and 5(d)]. Furthermore, looking more closely into the three spectra, the multiplet calculation seems to yield a better overlap with the experiment for Mn than the DFT method. In particular, the multiplet calculation succeeds in reproducing the positive shoulder on the high-energy side of the $L_{3}$ edge, while the DFT fails in doing so. In contrast, for Fe, there is a better agreement between the spectrum calculated from DFT and the experiment. Specifically, the ratio of the $L_{2} / L_{3}, \mathrm{XMCD}$ peaks are more consistent with DFT than CT calculations. In addition, for $\mathrm{Fe}$, the width of the peaks from multiplet calculation is too narrow in comparison with experiments and DFT calculation. This reduction in overall width of the peaks in multiplet calculation can be taken into account by a reduction of the Slater integrals [41]. The differences between $\mathrm{Mn}$ and $\mathrm{Fe}$ spectra suggest that although $\mathrm{Mn}$ and $\mathrm{Fe}$ atoms are closely related $3 d$ transition metals, they behave intrinsically differently in this system. More precisely, it is anticipated from previous studies that the Mn $3 d$ states occupy a more localized environment (the pyramidal $3 g$ site), while $\mathrm{Fe} 3 d$ states are in a more delocalized one (tetrahedron $3 f$ site) [42]. This may explain why the charge-transfer multiplet calculation cannot fully be implemented in the case of Fe.

To derive quantitative values for the spin and orbital moments $\left(\mu_{\text {spin }}\right.$ and $\left.\mu_{\text {orb }}\right)$ from XMCD spectra, sum rules are usually applied [43,44]. However, in the present case, some care has to be taken, especially for Mn [45-49]. Several issues neglected by the sum rule approach play a significant role. First of all, the relatively strong $2 p-3 d$ (core-valence) Coulomb interaction compared to $2 p$ spin-orbit interaction leads to mixing of the $j=2 p_{3 / 2}$ and $j=2 p_{1 / 2}$ manifolds, which consequently causes an inaccuracy for the integration over the spin-orbit split core levels. This feature is illustrated by the positive XMCD signal on the high-energy wing of $L_{3}$. The spin sum rule is only valid in the limit of $j j$ coupling and in the present case should be thus corrected for $j j$ mixing. Second, according to the sum rules, $\mu_{\text {spin }}$ and $\mu_{\text {orb }}$ depend on the number of valence holes in the $3 d$ state and a proportionality constant, the integrated area of magnetization-averaged signal. The former can be determined via theoretical calculations, but the latter requires care to obtain background subtraction and accurate edge steps, which often causes inaccuracies. Finally, the sum rules are often applied by omitting the magnetic dipole operator $T_{\mathrm{z}}$. For the present case, the $T_{\mathrm{z}}$ values are included but found to be quite small. Band structure calculations give 0.00098 and -0.00338 for $\mathrm{Mn}$ and Fe, respectively [50]. Here, we assumed the number of unoccupied $d$ holes to be 5 for $\mathrm{Mn}$ and 4 for $\mathrm{Fe}$. Following the procedure used originally by Chen et al. [51], we employed a simple two-step function to subtract the $L_{3}$ and $L_{2}$ edge steps from the absorption spectrum. Then by applying orbital sum rules [43], the orbital magnetic moments of $\mathrm{Mn}$ and $\mathrm{Fe}$ of compositions $A$ and $B$ are obtained and presented in Table I. A small positive orbital moment is observed for both $\mathrm{Mn}$ and $\mathrm{Fe}$, which confirms that the spin and orbital moments are coupled in parallel. Besides, the small nonzero value also indicates that $\mathrm{Mn}$ and $\mathrm{Fe}$ are not in a pure $3 d^{5}$ and $3 d^{6}$ ground state configuration, but have small admixtures of $3 d^{6}$ for $\mathrm{Mn}$ and $3 d^{7}$ for $\mathrm{Fe}$ due to the hybridization with neighboring atoms $[52,53]$. Though both the $\mathrm{Mn}$ and $\mathrm{Fe}$ atoms have a very small orbital magnetic 
TABLE I. The spin and orbital magnetic moments (in units of $\mu_{\mathrm{B}}$ /atom) derived from the sum rules and corrected spin-moment values are summarized along with the total magnetic moment obtained by SQUID magnetometry, for $\mathrm{MnFe}_{0.95} \mathrm{P}_{0.582} \mathrm{~B}_{0.078} \mathrm{Si}_{0.34}$ (composition $A$ ) and $\mathrm{Mn}_{1.25} \mathrm{Fe}_{0.70} \mathrm{P}_{0.50} \mathrm{~B}_{0.01} \mathrm{Si}_{0.49}$ (composition $B$ ) at $250 \mathrm{~K}$ (ferromagnetic state) and $330 \mathrm{~K}$ (paramagnetic state) in a field of $2 \mathrm{~T}$.

\begin{tabular}{|c|c|c|c|c|c|c|c|c|c|c|c|c|c|}
\hline \multirow[b]{3}{*}{ Composition } & \multirow[b]{3}{*}{ Atom } & \multicolumn{4}{|c|}{ Ferromagnetic $T=250 \mathrm{~K}$} & \multicolumn{4}{|c|}{ Paramagnetic $T=330 \mathrm{~K}$} & \multicolumn{2}{|c|}{ XMCD } & \multicolumn{2}{|c|}{$\mu$ (SQUID) } \\
\hline & & \multicolumn{2}{|c|}{$\mu_{\text {spin }}$} & \multirow{2}{*}{$\begin{array}{l}\mu_{\mathrm{orb}} \\
(\mathrm{SR})\end{array}$} & \multirow{2}{*}{$\begin{array}{c}\mu_{\text {orb }} / \mu_{\text {spin }} \\
\text { (Corr.) }\end{array}$} & \multicolumn{2}{|c|}{$\mu_{\text {spin }}$} & \multirow{2}{*}{$\begin{array}{l}\mu_{\text {orb }} \\
(\mathrm{SR})\end{array}$} & \multirow{2}{*}{$\begin{array}{c}\mu_{\text {orb }} / \mu_{\text {spin }} \\
\text { (Corr.) }\end{array}$} & \multirow[b]{2}{*}{$250 \mathrm{~K}$} & \multirow[b]{2}{*}{$330 \mathrm{~K}$} & \multirow[b]{2}{*}{$250 \mathrm{~K}$} & \multirow[b]{2}{*}{$330 \mathrm{~K}$} \\
\hline & & (SR) & (Corr.) & & & (SR) & (Corr.) & & & & & & \\
\hline \multirow{2}{*}{$A$} & $\langle\mathrm{Fe}\rangle 3 f$ & 0.671 & 0.804 & 0.046 & 0.057 & 0.190 & 0.235 & 0.012 & 0.051 & \multirow{2}{*}{2.032} & \multirow{2}{*}{0.555} & \multirow{2}{*}{2.96} & \multirow{2}{*}{0.37} \\
\hline & $\langle\mathrm{Mn}\rangle 3 g$ & 0.816 & 1.181 & 0.001 & 0.001 & 0.214 & 0.307 & 0.001 & 0.003 & & & & \\
\hline \multirow{2}{*}{$B$} & $\langle\mathrm{Fe}\rangle 3 f$ & 0.658 & 0.788 & 0.023 & 0.029 & 0.120 & 0.186 & 0.015 & 0.081 & \multirow{2}{*}{1.886} & \multirow{2}{*}{0.453} & \multirow{2}{*}{2.68} & \multirow{2}{*}{0.31} \\
\hline & $\langle\mathrm{Mn}\rangle 3 f+3 g$ & 0.739 & 1.069 & 0.006 & 0.006 & 0.172 & 0.247 & 0.005 & 0.020 & & & & \\
\hline
\end{tabular}

moment, it is interesting to note that $\mu_{\text {orb }}$ for $\mathrm{Fe}$ is one order of magnitude higher than for Mn.

To obtain more reliable spin moments, a correction procedure is used [45]. First, an experimental spin moment is derived from the sum rules [44]. Then, XAS and XMCD are simulated with charge-transfer multiplet calculations by fitting the experimental data. At the end, the expected spin moments are calculated for the ground state. By comparing this value to the sum-rules result, correction factors [45] $\left[S E_{z}^{\text {sum }}\right] /\left\langle S_{z}\right\rangle$ of 0.69 for $\mathrm{Mn}$ and 0.85 for Fe are derived, and are subsequently applied to all sum-rule values. In Table I, the spin and orbital magnetic moments derived from the sum rules and the corrected values are summarized along with the total magnetic moment obtained by SQUID magnetometry. For composition $A$, we obtain an effective magnetic moment for $\mathrm{Mn}$ of $\mu(\mathrm{Mn})=\mu_{\mathrm{spin}}+\mu_{\mathrm{orb}}=1.182 \mu_{\mathrm{B}}$ and for the Fe atom of $\mu(\mathrm{Fe})=\mu_{\text {spin }}+\mu_{\text {orb }}=0.85 \mu_{\mathrm{B}}$ in the ferromagnetic state $(T=250 \mathrm{~K}, 2 \mathrm{~T})$. The total magnetic moment per formula unit results in $2.032 \mu_{\mathrm{B}}$, which is about $30 \%$ smaller than the magnetization determined by magnetometry of $2.96 \mu_{\mathrm{B}} /$ f.u. In the paramagnetic state $(T=330 \mathrm{~K}, 2 \mathrm{~T}), \mathrm{Mn}$ moments of $\mu(\mathrm{Mn})=\mu_{\text {spin }}+\mu_{\text {orb }}=0.308 \mu_{\mathrm{B}}$ and Fe moments of $\mu(\mathrm{Fe})=$ $\mu_{\text {spin }}+\mu_{\text {orb }}=0.247 \mu_{\mathrm{B}}$ are obtained. For composition $B$, a slightly lower magnetic moment is observed, which is in accordance with the magnetization data shown in Fig. 1.

To gain further insight into the thermal evolution of the magnetic properties across the FOMT, systematic XMCD measurements were performed as a function of the temperature and magnetic field. The results are presented in Figs. 6 and 7 for materials $A$ and $B$, respectively. In Fig. 6(a), the Mn and $\mathrm{Fe}$ magnetic moments for composition $A$ are shown as a function of temperature for a magnetic field of $2 \mathrm{~T}$. The most striking feature is the larger magnetic moments for $\mathrm{Mn}$ compared to $\mathrm{Fe}$. This is in line with previous neutron diffraction studies in the ferromagnetic phase $[18,22]$. This phenomenon was ascribed to the site occupancy, with $\mathrm{Mn}$ preferentially occupying the high moment $3 g$ site and Fe the $3 f$ site, which shows a weaker magnetism [18,22,54,55]. It is now found experimentally that this tendency is also maintained in the paramagnetic state, as was suggested by first-principles calculations [11]. If we now look at the relative evolution of the projected local moments for each element, we can observe that both exhibit an abrupt decrease at $T_{\mathrm{C}}$. From 250 to $330 \mathrm{~K}$, the reduction in magnetic moments is $72 \%$ and $74 \%$ for $\mathrm{Fe}$ and $\mathrm{Mn}$, respectively (note that this reduction is not significantly affected by the sum-rule correction). This thus points towards a similar evolution for the magnetism of the $\mathrm{Fe}$ and Mn moments across the FOMT. This trend is also found in the XMCD as a function of field. Figure 6(b) displays the magnetic field dependence of the XMCD of composition $A$ in the ferromagnetic and paramagnetic states as well as at $T_{\mathrm{C}}$ (in a field of $2 \mathrm{~T}$ ). At $250 \mathrm{~K}$, a field dependence characteristic for a ferromagnetic state is observed for both Fe and Mn. At $292 \mathrm{~K}$, the field-induced transition can clearly be seen for both $\mathrm{Mn}$ and $\mathrm{Fe}$. Although these inflection points are less pronounced than that observed from magnetization measurement [see Figs. 1(c) and 1(e)], it is still present. The transition is centered at a magnetic field of $2 \mathrm{~T}$. In the paramagnetic state, the application of a magnetic field increases the projected magnetic moments
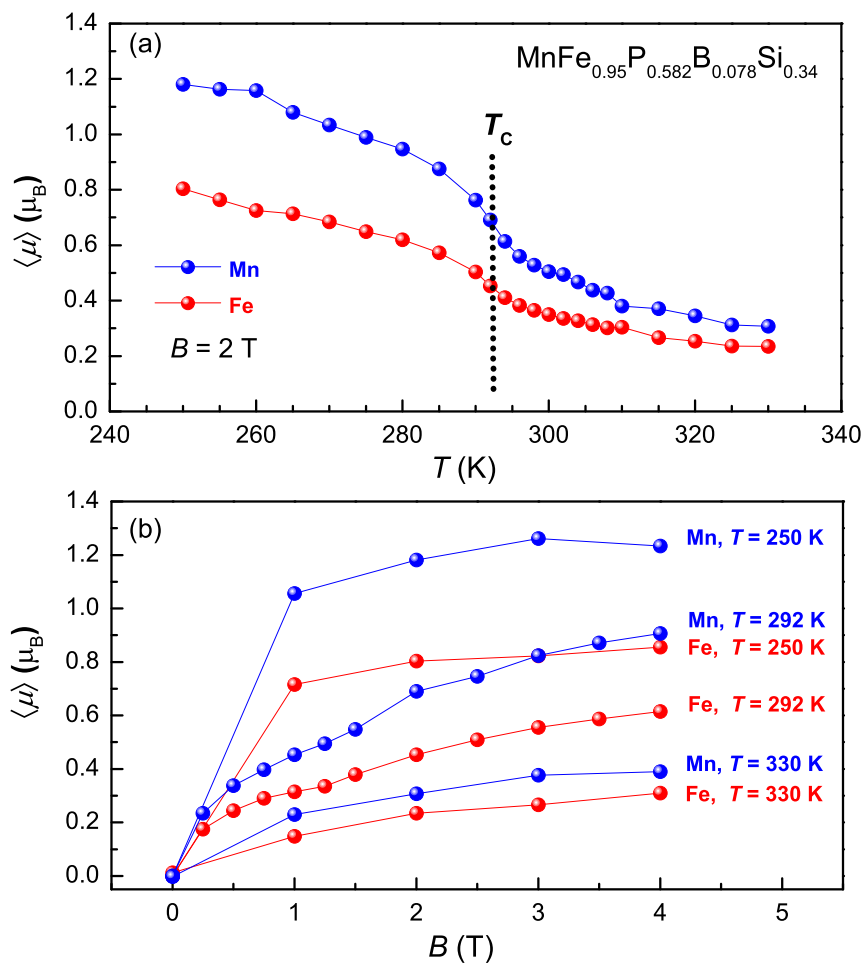

FIG. 6. (Color online) The XMCD magnetic moment as a function of temperature (a) and magnetic field (b) for $\mathrm{MnFe}_{0.95} \mathrm{P}_{0.582} \mathrm{~B}_{0.078} \mathrm{Si}_{0.34}$ (composition $A$ ). Top curves were measured in a field of $2 \mathrm{~T}$; below curves were measured at $250 \mathrm{~K}$ (ferromagnetic state), $292 \mathrm{~K}$ (near transition), and $330 \mathrm{~K}$ (paramagnetic state). The XMCD magnetic moments were derived as described in the text. 

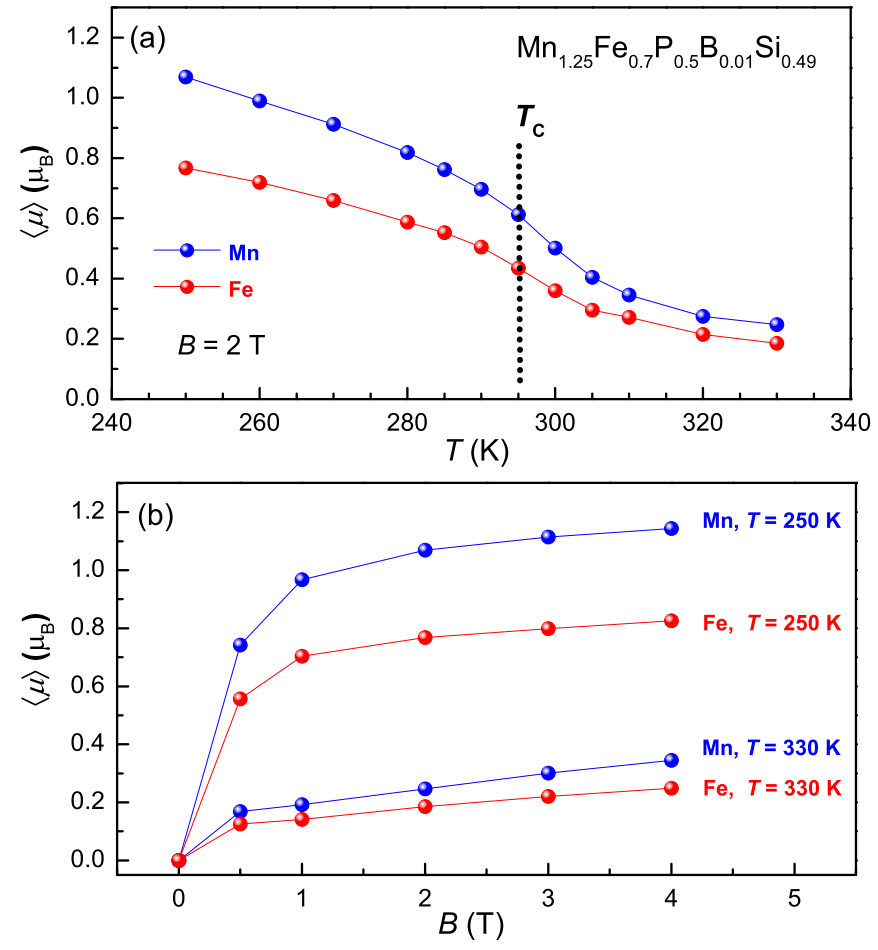

FIG. 7. (Color online) The XMCD magnetic moment as a function of temperature (a) and magnetic field (b) for $\mathrm{Mn}_{1.25} \mathrm{Fe}_{0.70} \mathrm{P}_{0.50} \mathrm{~B}_{0.01} \mathrm{Si}_{0.49}$ (composition $B$ ). Top curves were measured in a field of $2 \mathrm{~T}$; below curves were measured at $250 \mathrm{~K}$ (ferromagnetic state) and $330 \mathrm{~K}$ (paramagnetic state). The XMCD magnetic moments were derived as described in the text.

for both $\mathrm{Mn}$ and $\mathrm{Fe}$ at an identical rate $0.06(1) \mu_{\mathrm{B}} T^{-1}$. The XMCD versus $T$ and $B$ are consistent and both indicate that the Fe magnetic moment remains finite in the paramagnetic phase. The $T$ or $B$ evolution of the Fe moment is similar to that of Mn.

Let us now consider the case of composition $B$, which exhibits a transitional behavior lying at the FOMT and secondorder magnetic phase transition boundary. The XMCD as a function of temperature (at fixed field) and as a function of the magnetic field (at constant temperature) in the ferromagnetic and paramagnetic states is presented in Figs. 7(a) and 7(b), respectively. The total magnetization in the ferromagnetic state is lower in composition $B$ than in $A$. This result is in line with the previous reports in this system [56]. The Mn moments are expected to have a significantly lower magnetic moment on the $3 f$ site than on the $3 g$ site (and also lower than Fe on the same $3 f$ site) [11]. This theoretical prediction is here confirmed, since the decrease in the total magnetization by the change in $\mathrm{Mn} / \mathrm{Fe}$ ratio can be mainly ascribed to the reduction in $\mathrm{Mn}$ moment (the Mn moment is reduced more strongly than the Fe moment). Previous neutron experiments [18] provided the magnetic moment on $\mathrm{Mn}$ in the $3 g$ position (fully occupied by $\mathrm{Mn}$ ) and their evolution with a change in $\mathrm{Mn} / \mathrm{Fe}$ ratio, but failed to separate the $\mathrm{Mn} / \mathrm{Fe}$ magnetic moments on the $3 f$ site. The present XMCD approach allows one to obtain the Fe moment independently of the Mn signal and to estimate the Mn moment on the $3 f$ site. By considering that the Mn magnetic moments on the $3 g$ site are found to be independent of the $\mathrm{Mn} / \mathrm{Fe}$ ratio [18,22], one can derive for composition $B$ an experimental value for the Mn moments on the $3 f$ site of $\mu_{\mathrm{Mn}(3 f)}=\frac{1}{x}\left\langle\mu_{\mathrm{Mn}}\right\rangle-\frac{1-x}{x} \mu_{\mathrm{Mn}(3 g)}=0.621 \mu_{\mathrm{B}}$, where $x$ is the $\mathrm{Mn}(3 f) / \mathrm{Mn}$ ratio. It should be noted that (i) this moment is derived from XMCD data only and (ii) as observed for Fe moment, the Mn moment on the $3 f$ site is lower than the $3 g$ site.

If one looks at the temperature evolution of the $\mathrm{Mn} / \mathrm{Fe}$ moments for composition $B$, once again there is a striking similarity between the respective evolution of the two elements with a reduction from 250 to $330 \mathrm{~K}$ of $75 \%$ and $76 \%$ for $\mathrm{Fe}$ and $\mathrm{Mn}$, respectively. This $\mathrm{Fe} / \mathrm{Mn}$ comparative approach for composition $B$ is not straightforward as the signal for $\mathrm{Mn}$ mixes the temperature dependence of the $3 f$ and $3 g$ sites. The Mn moment on the $3 f$ position shows the same local moment quenching as predicted for Fe. The similarity of the Fe and Mn moments evolution, however, supports the overall similarity between the temperature dependence of the $3 f$ and $3 g$ sites observed for composition $A$, i.e., no complete quenching of the local moment on the $3 f$ site is observed for temperatures directly above the FOMT.

The observed similarity in temperature and field dependence for the $\mathrm{Mn}$ and Fe moments across the ferroparamagnetic transition does not support the quenching of the $3 f$ magnetic moment for $\mathrm{Fe}$ in the paramagnetic state, predicted from the electronic structure calculations on $\mathrm{MnFeP}_{0.5} \mathrm{Si}_{0.5}$ [11]. These XMCD results therefore suggest that the reduction in the $3 f$ moments at $T_{\mathrm{C}}$ is overestimated by $a b$ initio calculations. This observation does not, however, directly affect the predicted reconstruction of the electronic state at the $3 f$ site, as the reconstruction was also predicted for the parent phase $\mathrm{Fe}_{2} \mathrm{P}$, without a full moment quenching of the $3 f$ site [24].

Possible origins for this difference are the limited size of the supercell used in the calculations and the effect of shortrange correlations as, strictly speaking, the electronic structure calculations do not take into account the effect of temperature. In the XMCD experiments, the temperatures considered are $T_{\mathrm{C}} \pm 40 \mathrm{~K}$. This temperature range is significantly larger than the purely discontinuous regime at the FOMT and its corresponding magnetic discontinuity. However, one is still in the transition range where short-range order develops $[17,18,57,58]$. In the paramagnetic phase, the $M_{\mathrm{T}}(B)$ curves of the XMCD magnetic moments show in the low magnetic field regime a deviation from Curie-Weiss behavior, indicating the existence of short-range order with temporal fluctuations of ferromagnetically ordered clusters. The existence of shortrange magnetic order in the paramagnetic phase has been reported in various $\mathrm{Fe}_{2} \mathrm{P}$-based materials up to $3 T_{\mathrm{C}}$ [18,57]. These fluctuations above the transition may contribute to a smearing of the $\operatorname{Fe}(3 f)$ moment quenching.

\section{CONCLUSIONS}

The electronic and magnetic properties of $(\mathrm{Mn}, \mathrm{Fe})_{2}(\mathrm{P}, \mathrm{Si}, \mathrm{B})$ materials across their first-order magnetic phase transition have been investigated in an element-specific way by XMCD measurements. The results are compared with CT multiplet and DFT calculations. From XAS, it is found that no significant valence change and generally speaking no spectral shape modification is observed across the FOMT. 
From XMCD, the magnetic field and temperature dependence of the magnetic moments was obtained for the $\mathrm{Fe}$ and $\mathrm{Mn}$ moments for two $\mathrm{Fe} / \mathrm{Mn}$ ratios. It is observed that the $\mathrm{Mn}$ exhibits a much lower magnetization on the $3 f$ site than on the $3 g$. In contrast to theoretical predictions, it is observed that the Fe moments are not fully quenched in the paramagnetic state just above $T_{\mathrm{C}}$. These results indicate that the magnitude of the reduction in the $3 f$ moments at $T_{\mathrm{C}}$ is overestimated by ab initio calculations.

\section{ACKNOWLEDGMENTS}

We acknowledge the European Synchrotron Radiation Facility for provision of synchrotron radiation facilities and thank the beamline staff for assistance in using beam line ID08. The authors would like to thank Cinthia Piamonteze and Anne-Christine Uldry for helpful discussions. This work is financially supported by the Foundation for Fundamental Research on Matter (FOM) (The Netherlands) and BASF New Business.
[1] A. M. Tishin and Y. I. Spichkin, The Magnetocaloric Effect and its Applications (Institute of Physics Publishing, Bristol, 2003).

[2] K. A. Gschneidner, Jr., V. K. Pecharsky, and A. O. Tsokol, Rep. Prog. Phys. 68, 1479 (2005).

[3] A. Smith, C. R. Bahl, R. Bjørk, K. Engelbrecht, K. K. Nielsen, and N. Pryds, Adv. Energy Mater. 2, 1288 (2012).

[4] M. P. Annaorazov, S. A. Nikitin, A. L. Tyurin, K. A. Asatryan, and A. K. Dovletov, J. Appl. Phys. 79, 1689 (1996).

[5] V. K. Pecharsky and K. A. Gschneidner, Jr., Phys. Rev. Lett. 78, 4494 (1997).

[6] H. Wada and Y. Tanabe, Appl. Phys. Lett. 79, 3302 (2001).

[7] F.-X. Hu, B.-G. Shen, J.-R. Sun, Z.-H. Cheng, G.-H. Rao, and X.-X. Zhang, Appl. Phys. Lett. 78, 3675 (2001).

[8] A. Fujita, S. Fujieda, Y. Hasegawa, and K. Fukamichi, Phys. Rev. B 67, 104416 (2003).

[9] O. Tegus, E. Brück, K. H. J. Buschow, and F. R. de Boer, Nature (London) 415, 150 (2002).

[10] N. T. Trung, L. Zhang, L. Caron, K. H. J. Buschow, and E. Brück, Appl. Phys. Lett. 96, 172504 (2010).

[11] N. H. Dung, Z. Q. Ou, L. Caron, L. Zhang, D. T. C. Thanh, G. A. de Wijs, R. A. de Groot, K. H. J. Buschow, and E. Brück, Adv. Energy Mater. 1, 1215 (2011).

[12] H. Yibole, F. Guillou, L. Zhang, N. H. van Dijk, and E. Brück, J. Phys. D: Appl. Phys. 47, 075002 (2014).

[13] F. Guillou, G. Porcari, H. Yibole, N. van Dijk, and E. Brück, Adv. Mater. 26, 2671 (2014).

[14] V. K. Pecharsky, K. A. Gschneidner, A. O. Pecharsky, and A. M. Tishin, Phys. Rev. B 64, 144406 (2001).

[15] M. Annaorazov, K. Bärner, and Ş. Yalçin, J. Alloys Compd. 372, 52 (2004).

[16] K. G. Sandeman, Scr. Mater. 67, 566 (2012).

[17] O. Beckman and L. Lundgren, Handbook of Magnetic Materials (North-Holland, Amsterdam, 1991).

[18] N. H. Dung, L. Zhang, Z. Q. Ou, L. Zhao, L. van Eijck, A. M. Mulders, M. Avdeev, E. Suard, N. H. van Dijk, and E. Brück, Phys. Rev. B 86, 045134 (2012).

[19] L. Zhang, O. Može, K. Prokeš, O. Tegus, and E. Brück, J. Magn. Magn. Mater. 290, 679 (2005).

[20] D. M. Liu, Q. Z. Huang, M. Yue, J. W. Lynn, L. J. Liu, Y. Chen, Z. H. Wu, and J. X. Zhang, Phys. Rev. B 80, 174415 (2009).

[21] V. Höglin, M. Hudl, M. Sahlberg, P. Nordblad, P. Beran, and Y. Andersson, J. Solid State Chem. 184, 2434 (2011).

[22] Z. Ou, L. Zhang, N. Dung, L. van Eijck, A. Mulders, M. Avdeev, N. van Dijk, and E. Brück, J. Magn. Magn. Mater. 340, 80 (2013).

[23] M. Yue, D. M. Liu, Q. Z. Huang, T. Wang, F. X. Hu, J. B. Li, G. H. Rao, B. G. Shen, J. W. Lynn, and J. X. Zhang, J. Appl. Phys. 113, 043925 (2013).
[24] H. Yamada and K. Terao, Phase Transitions 75, 231 (2002).

[25] Z. Gercsi, E. K. Delczeg-Czirjak, L. Vitos, A. S. Wills, A. Daoud-Aladine, and K. G. Sandeman, Phys. Rev. B 88, 024417 (2013).

[26] N. H. Dung, L. Zhang, Z. Q. Ou, and E. Brück, Scr. Mater. 67, 975 (2012).

[27] F. M. F. de Groot, J. C. Fuggle, B. T. Thole, and G. A. Sawatzky, Phys. Rev. B 42, 5459 (1990).

[28] F. de Groot, Coord. Chem. Rev. 249, 31 (2005).

[29] E. Stavitski and F. M. de Groot, Micron 41, 687 (2010).

[30] P. Blaha, K. Schwarz, G. Madsen, D. Kvasnicka, and J. Luitz, WIEN2k (TU Wien, Austria, 2001).

[31] D. Singh, Planewaves, Pseudopotentials and the LAPW Method (Kluwer Academic, London, 1994).

[32] J. P. Perdew, K. Burke, and M. Ernzerhof, Phys. Rev. Lett. 77, 3865 (1996).

[33] C. Ambrosch-Draxl and J. O. Sofo, Comput. Phys. Commun. 175, 1 (2006).

[34] K. W. Edmonds, G. van der Laan, A. A. Freeman, N. R. S. Farley, T. K. Johal, R. P. Campion, C. T. Foxon, B. L. Gallagher, and E. Arenholz, Phys. Rev. Lett. 96, 117207 (2006).

[35] P. Klaer, B. Balke, V. Alijani, J. Winterlik, G. H. Fecher, C. Felser, and H. J. Elmers, Phys. Rev. B 84, 144413 (2011).

[36] K. R. Priolkar, P. A. Bhobe, D. N. Lobo, S. W. D’Souza, S. R. Barman, A. Chakrabarti, and S. Emura, Phys. Rev. B 87, 144412 (2013).

[37] H. A. Dürr, G. van der Laan, D. Spanke, F. U. Hillebrecht, and N. B. Brookes, Phys. Rev. B 56, 8156 (1997).

[38] L. Caron, M. Hudl, V. Höglin, N. H. Dung, C. P. Gomez, M. Sahlberg, E. Brück, Y. Andersson, and P. Nordblad, Phys. Rev. B 88, 094440 (2013).

[39] G. van der Laan and B. T. Thole, Phys. Rev. B 43, 13401 (1991).

[40] J. Zaanen, G. A. Sawatzky, and J. W. Allen, Phys. Rev. Lett. 55, 418 (1985).

[41] G. van der Laan, J. Zaanen, G. A. Sawatzky, R. Karnatak, and J.-M. Esteva, Phys. Rev. B 33, 4253 (1986).

[42] C. Jian-Wang, L. He-Lie, and Z. Qing-Qi, J. Phys.: Condens. Matter 5, 9307 (1993).

[43] B. T. Thole, P. Carra, F. Sette, and G. van der Laan, Phys. Rev. Lett. 68, 1943 (1992).

[44] P. Carra, B. T. Thole, M. Altarelli, and X. Wang, Phys. Rev. Lett. 70, 694 (1993).

[45] C. Piamonteze, P. Miedema, and F. M. F. de Groot, Phys. Rev. B 80, 184410 (2009).

[46] Y. Teramura, A. Tanaka, and T. Jo, J. Phys. Soc. Jpn. 65, 1053 (1996). 
[47] G. van der Laan, K. T. Moore, J. G. Tobin, B. W. Chung, M. A. Wall, and A. J. Schwartz, Phys. Rev. Lett. 93, 097401 (2004).

[48] R. Wu, D. Wang, and A. J. Freeman, Phys. Rev. Lett. 71, 3581 (1993).

[49] J. P. Crocombette, B. T. Thole, and F. Jollet, J. Phys.: Condens. Matter 8, 4095 (1996).

[50] A. Perlov, A. Yaresko, and V. Antonov, Spin-polarized relativistic linear muffin-tin orbitals package for electronic structure calculations, PY-LMTO (unpublished).

[51] C. T. Chen, Y. U. Idzerda, H.-J. Lin, N. V. Smith, G. Meigs, E. Chaban, G. H. Ho, E. Pellegrin, and F. Sette, Phys. Rev. Lett. 75, 152 (1995).

[52] G. van der Laan, K. W. Edmonds, E. Arenholz, N. R. S. Farley, and B. L. Gallagher, Phys. Rev. B 81, 214422 (2010).
[53] F. Kronast, R. Ovsyannikov, A. Vollmer, H. A. Dürr, W. Eberhardt, P. Imperia, D. Schmitz, G. M. Schott, C. Ruester, C. Gould, G. Schmidt, K. Brunner, M. Sawicki, and L. W. Molenkamp, Phys. Rev. B 74, 235213 (2006).

[54] X. B. Liu and Z. Altounian, J. Appl. Phys. 105, 07A902 (2009).

[55] M. Hudl, P. Nordblad, T. Björkman, O. Eriksson, L. Häggström, M. Sahlberg, Y. Andersson, E.-K. Delczeg-Czirjak, and L. Vitos, Phys. Rev. B 83, 134420 (2011).

[56] F. Guillou, H. Yibole, G. Porcari, L. Zhang, N. H. van Dijk, and E. Brück, J. Appl. Phys. 116, 063903 (2014).

[57] H. Fujii, Y. Uwatoko, K. Motoya, Y. Ito, and T. Okamoto, J. Phys. Soc. Jpn. 57, 2143 (1988).

[58] R. Zach, M. Guillot, and R. Fruchart, J. Magn. Magn. Mater. 89, 221 (1990). 